\title{
SiON integrated optics elliptic couplers for Fizeau-based Optical Coherence Tomography
}

\author{
V. Duc Nguyen, T.G. van Leeuwen, and J. Kalkman \\ Biomedical Engineering \& Physics, \\ Academic Medical Center, University of Amsterdam \\ Amsterdam, The Netherlands \\ D.V.Nguyen@amc.uva.nl
}

\begin{abstract}
The use of integrated optics for Optical Coherence Tomography (OCT) can offer significant cost reductions and new applications. We designed, fabricated, and characterized Silicon oxynitride ( $\mathrm{SiON}$ ) elliptic couplers that are used to focus light from a chip into the off-chip environment. Fizeau-based OCT measurements are performed and compared to calculations.
\end{abstract}

Keywords- optical coherence tomography; integrated optics; elliptic coupler;

\section{INTRODUCTION}

Optical Coherence Tomography (OCT) [1] is a noninvasive optical technique for imaging biological tissue to a few millimeters depth with micrometer resolution. OCT is gaining widespread use in the clinic, in particular its use in ophthalmology for imaging the retina is becoming routine clinical practice. OCT uses interferometry to detect the timeof-flight of light reflected from a tissue. Nowadays, most OCT systems are Fourier-domain OCT systems, measuring the interference spectrum, formed by the reflection of a reference arm and biological tissue, by using a spectrometer or a swept source. Fourier-domain OCT systems are composed of many bulk optical components, such as: optical fibers, (focusing) lenses, beam splitters, and galvanometric scanners that all are necessary to relay the optical signal through the OCT system. The combination of all these components makes OCT systems bulky, expensive, and complex. Integrated optics can integrate these bulk components that make an OCT system onto a single optical chip. Therefore, integrated optics can make OCT systems smaller and more cost efficient. Thus far, only work has been presented about the integration of beamsplitters and waveguides in integrated optics for OCT [2], however many other OCT components also can be integrated. In addition, integrated optics can offer additional OCT functionality that would be too difficult or complex to achieve with bulk optics.

One key OCT component is a sample arm focusing lens. Here we present integrated optics elliptic couplers designed and fabricated in Silicon oxynitride ( $\mathrm{SiON}$ ) as an integratedoptics solution to focus light from the chip to the off-chip environment.

\author{
N. Ismail, F. Sun, B. I. Akca, A. Driessen, M. Pollnau, \\ R. M. de Ridder, and K. Wörhoff \\ Integrated Optical Microsystems Group, \\ MESA+ Institute for Nanotechnology, University of Twente \\ Enschede, The Netherlands
}

\section{ELLIPTIC COUPLERS}

An integrated optics elliptic coupler consists of a straight index guiding waveguide followed by one half of an ellipticshaped index guiding section [3], as shown in Fig.1. The elliptic coupler ends in an air region where sensing in the offchip environment can take place. In a ray optics view, all rays emanating from one focal point of an ellipse are imaged onto the second focal point. Since the straight waveguide exits close to the first focal point, and it is thus to be expected that many rays will be imaged close to the second focal point. Some of the rays exiting the straight waveguide are not reflected on the boundary of the elliptic coupler and propagate without being focused into the air region.

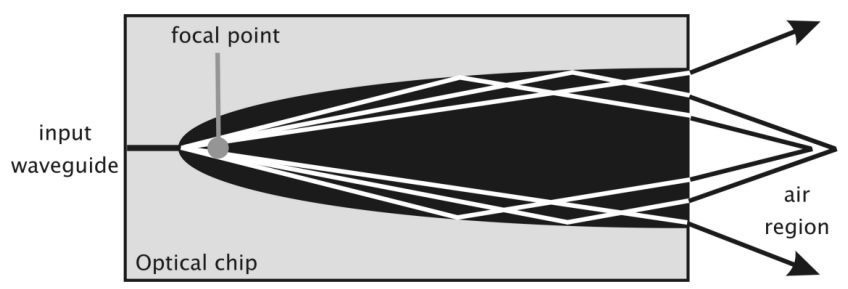

Fig. 1. Integrated optics elliptic coupler geometry. Rays from the input waveguide reflect off the elliptic coupler edge and are focused in the air region.

\section{MATERIALS AND METHODS}

We designed and fabricated straight waveguides/elliptic couplers in SiON technology [4]. The SiON layer has a thickness of $820 \mathrm{~nm}(\mathrm{n}=1.5337)$ and is surrounded by $\mathrm{SiO}_{2}$. For single mode operation we designed the straight waveguides with $2.5 \mu \mathrm{m}$ width.

The electric field in the air region is determined in two ways. First, we perform beam propagation method (BPM) simulations of the electric field in the plane of the chip. Second, the beam profile intensity is measured in the plane of the chip using a knife-edge set-up and an infrared power meter. Finally, the field exiting from the elliptic coupler is modeled by Gaussian optics using the simulated and measured beam waists.

This work is supported by the Dutch Smartmix-Memphis project.. The authors gratefully acknowledge the technical support by G. Sengo. 


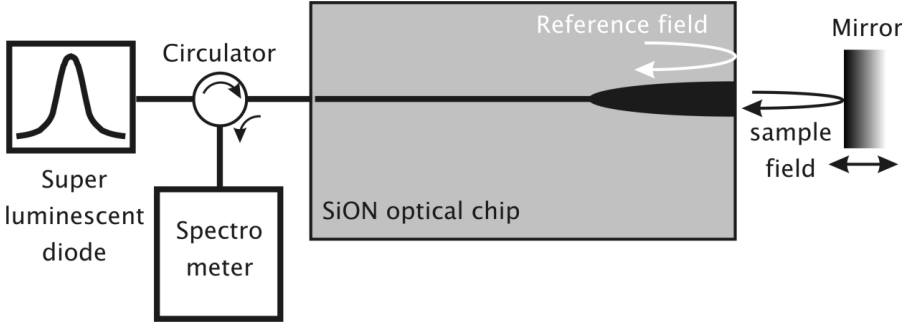

Fig. 2. Fizeau-based spectral-domain OCT set-up. Broadband light is coupled into the elliptic coupler and light reflected from the endfacet and the mirror is directed through the circulator into the spectrometer. The beam profiles are measured with a knife edge at the exit of the elliptic coupler.

Interferometric measurements are performed using a common path Fizeau-based OCT measurements, see Fig. 2. In short: light from a broadband lightsource operating at $1300 \mathrm{~nm}$ wavelength is coupled through a circulator into a tapered fiber. Via the tapered fiber light is coupled into waveguides with elliptic couplers at the other end of the optical chip. The field is reflected on the end facet of the elliptic coupler and on a mirror that is mounted on a moveable stage. The reflected light is guided through the circulator to a spectrometer where it is detected. Due to interference of the two fields, the spectrum is modulated and a depth profile is calculated by taking the Fourier transform of the spectrum. After a correction of the OCT signal for the intrinsic OCT system sensitivity with depth the OCT signal is only determined by the field evolution in the air region.

\section{OCT MEASUREMENTS}

Figure 3 and 4 show the results for an elliptic coupler with a semimajor axis length of $1000 \mu \mathrm{m}$ and a semiminor axis length of $35 \mu \mathrm{m}$. Figure 3 shows the beam waist for various distances from the end facet (intensity at $1 / \mathrm{e}^{2}$ level) measured in the plane of the chip with the knife-edge method. It can be clearly observed that the beam is focused at a distance of 500 $\mu \mathrm{m}$ and that the measured beam waists are in good agreement with the BPM simulations. In the out of plane direction the beam is strongly divergent.

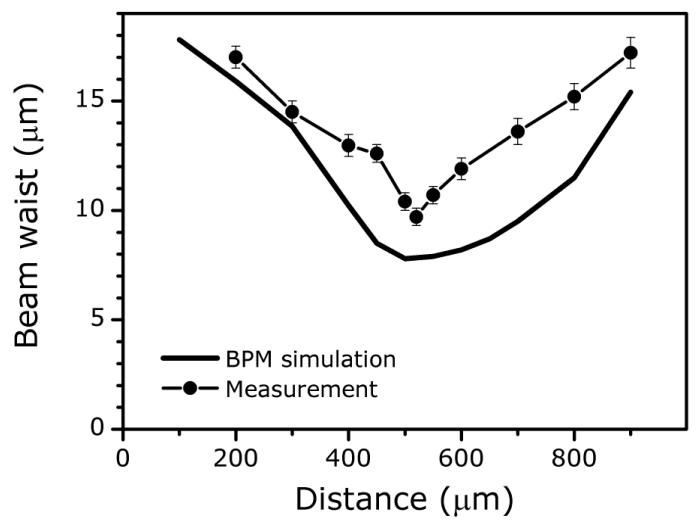

Fig. 3 Beam profile measurements in the plane of the chip as a function of distance for an elliptic coupler with semimajor axis length of $1000 \mu \mathrm{m}$ and a semiminor axis length of $35 \mu \mathrm{m}$ (dots). The experimental results are compared to BPM simulations (solid line)

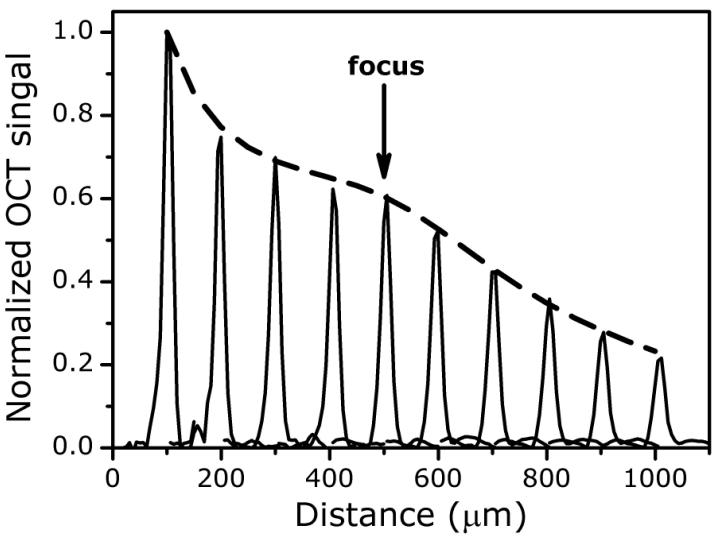

Fig. 4. Fizeau-based OCT depth profiles for a mirror at various distances from the end facet. The measurements for the various distances are superimposed on top of each other. The dashed line shows the decrease of the signal in depth calculated from the measured beam waist (see Fig. 3).

Figure 4 shows several OCT depth profile measurements overlaid on top of each other for various distances of the mirror relative to the end facet. The signal is normalized to the signal at $100 \mu \mathrm{m}$ depth. As can be observed, the OCT signal strength decreases with depth, which is primarily caused by the divergent beam in the out of plane direction. In the in-plane direction the elliptic coupler focuses the beam at a distance indicated by the arrow. Gaussian optics calculations that are based on the measured beam waist are overlaid on the data (dashed line). Good agreement between the measurement and the calculations is observed.

\section{DisCUSSION/CONCLUSIONS}

We characterized several elliptic couplers. All show good focusing properties in the in-plane direction. Fizeau-based OCT measurements show good agreement with calculations for all elliptic couplers. Compared to a straight waveguide elliptic couplers show a clear increase of the signal with depth. The strong decrease of the OCT signal with depth is mainly due to the strong divergence in the out-of-plane direction where no focusing takes place. The strong out-of-plane divergence is caused by the small height of the waveguide resulting in a strongly divergent field. We propose tapering of $\mathrm{SiON}$ waveguides to decrease the out-of-plane divergence of these elliptic couplers.

\section{REFERENCES}

[1] D. Huang et al., "Optical coherence tomography" Science 254, 1178 (1991)

[2] D. Culemann, A. Knuettel, and E. Voges, "Integrated optical sensor in glass for optical coherence tomography", IEEE J. Select. Topics Quantum Electron. 5, 730 ( 2000)

[3] C. Wei, F. Groen, M. K. Smit, I. Moerman, P.Van Daele, and R. Baets, "Integrated opical elliptic couplers: modeling, design, and applications," J. Lightwave Techol. I 15, 906 (1997)

[4] K. Wörhoff, C. G. K. Roeloffzen, R. M. de Ridder, A. Driessen, and P.V. Lambeck, "Design and application of compact and highly tolerant polarization-independent waveguides," J. Lightwave Technol. 25, 1276 (2007) 